\title{
miR-497/MIR497HG inhibits glioma cell proliferation by targeting CCNE1 and the miR-588/TUSC1 axis
}

\author{
LI-YA JI $^{1,2^{*}}$, MING WEI $^{3,4^{*}}$, YUAN-YUAN LIU ${ }^{3}$, ZHENG-LI DI $^{1}$ and SAN-ZHONG LI ${ }^{2}$ \\ ${ }^{1}$ Department of Neurology, Xi'an Central Hospital, Xi'an Jiaotong University, Xi'an, Shaanxi 710054; \\ ${ }^{2}$ Department of Neurosurgery, Xijing Hospital, Fourth Military Medical University, Xi'an, Shaanxi 710032; \\ ${ }^{3}$ Department of Neurology, Weishou Hospital of Luoyang; ${ }^{4}$ Department of Surgery, \\ No. 989 Hospital of People's Liberation Army, Luoyang, Henan 471000, P.R. China
}

Received May 6, 2021; Accepted September 6, 2021

DOI: $10.3892 / o r .2021 .8206$

\begin{abstract}
Emerging evidence has shown that microRNA (miR)-497 serves pivotal roles in tumorigenesis, cancer progression, metastasis and chemotherapy resistance in several types of cancer. In the present study, the expression and biological functions of miR-497 host gene (MIR497HG) were investigated in glioma tissue. The expression levels of miR-497 and MIR497HG were measured in glioma, adjacent non-cancerous and normal brain tissue and their association with the prognosis of patients with glioma were analyzed. The biological roles of miR-497 and MIR497HG were investigated in glioma cell lines. In addition, bioinformatics analysis, luciferase reporter assay and functional experiments were performed to identify and validate the downstream targets of miR-497 or MIR497HG. The expression levels of miR-497 and MIR497HG were downregulated in glioma tissue and cell lines compared with those in adjacent non-cancerous and normal brain tissue and normal human cortical neuron cell line. Patients with low miR-497 or MIR497HG expression levels exhibited a poor prognostic outcome. In addition, forced overexpression of miR-497 or MIR497HG significantly inhibited the proliferation and cell cycle progression of glioma cell lines. Furthermore, the results indicated that miR-497 and MIR497HG exerted their biological functions by direct targeting of cyclin E1 and miR-588/tumor suppressor
\end{abstract}

Correspondence to: Professor San-Zhong Li, Department of Neurosurgery, Xijing Hospital, Fourth Military Medical University, 169 Chang-Le Xi Street, Xi'an, Shaanxi 710032, P.R. China

E-mail: sunny_3c@126.com

Professor Zheng-Li Di, Department of Neurology, Xi'an Central Hospital, Xi'an Jiaotong University, 161 Xi-Wu Street, Xi'an, Shaanxi 710054, P.R. China

E-mail: zhenglidi@126.com

*Contributed equally

Key words: miR-497, MIR497HG, glioma, cyclin E1, miR-588, tumor suppressor candidate 1 candidate 1 . In summary, the data indicated that miR-497 and MIR497HG served as tumor suppressors and may be used as potential therapeutic targets and prognostic biomarkers in glioma.

\section{Introduction}

Neoplasms in the brain and other parts of the nervous system are the leading cause of cancer-associated mortality among men aged $<40$ years and women aged $<20$ years (1). These tumor types were estimated to cause 17,760 deaths in the US in 2019 (1). Gliomas account for $>70 \%$ of all primary brain tumors in adults and the majority of patients with glioma will not survive beyond the first 2 years from diagnosis even following aggressive chemotherapy or radiotherapy (2). Therefore, it is urgent to identify novel therapeutic methods to treat this disease. Although the molecular mechanisms that contribute to tumorigenesis of glioma have been recently identified (3), their exact association with the development of this disease has not been fully clarified.

MicroRNAs (miRs) are small, non-coding (nc), single-stranded RNAs that are 20-23 nucleotides in length and act by suppressing gene expression in a variety of eukaryotic organisms via targeting specific mRNAs (4). miRs suppress gene expression via complementary binding between their seed region and the 3'-untranslated region (UTR) of the target mRNA (4). Through this regulation, miRs serve a pivotal role in several cellular processes, including proliferation, cell cycle control, programmed cell death, differentiation, invasiveness and tissue specific functions, such as immune response, hormone secretion and angiogenesis, which are implicated in the development and progression of hepatocellular carcinoma (5), glioma (6) and lung cancer (7). miRs can be used as diagnostic and prognostic markers (8) in cancer as they are regulatory molecules with both oncogene and suppressor gene functions and constitute major components of intercellular communication (9). It has been shown that miRs are involved in several biological processes in glioma, such as cell proliferation, invasion, angiogenesis and therapeutic resistance (10). Significantly decreased expression of miR-49(6)7 has been observed in numerous types of cancer type, including cutaneous squamous 
cell carcinoma (11), breast (12), gastric (13), thyroid (14), colorectal (15), hepatocellular carcinoma (16), pancreatic (17), adrenocortical carcinoma (18), bladder (19), non-small cell lung (20) and cervical cancer (21) as well as other solid tumors. However, several studies have shown that miR-497 expression is upregulated in other types of cancer, including chronic lymphocytic leukemia (22), diffuse large B-cell lymphoma (23) and renal cell carcinoma (24). With regards to glioma, the expression and biological functions of miR-497 remain controversial and require further analysis. miR-497 is located at the intron of miR-497 host gene (MIR497HG), which has been demonstrated to be a tumor suppressor gene that suppresses tumor progression $(25,26)$. However, it remains controversial whether the long (1)nc RNA MIR497HG is the actual host gene of miR-497. In the present study, the expression and functions of miR-497 and its host gene MIR497HG were investigated in glioma tissue.

\section{Materials and methods}

Sample collection and ethics statement. All human tissue was obtained from patients undergoing surgery for glioma in the Department of Neurosurgery, Xijing Hospital, Fourth Military Medical University (Xi'an, China). A total of 13 male and 17 female glioma patients aged 40-60 years were recruited between January 2018 and January 2020. The glioma samples were histologically classified based on the diagnosis of clinical and pathological grading according to the World Health Organization guidelines (27). Among these samples, 22 contained both adjacent and glioma tissues, while 8 samples only contained glioma tissue. Therefore, the number of glioma tissues was 30 and the number of adjacent tumor tissues was 22. The normal brain tissue was collected as negative controls from patients with cerebral trauma. Written informed consent conforming to the tenets of the Declaration of Helsinki was obtained from each participant and the study procedures were approved by the institutional review board of Xijing Hospital, Fourth Military Medical University. Expression levels of CCNE1 and TUSC1 correlated with overall survival time were analyzed using 667 patients derived from The Cancer Genome Atlas (TCGA) database (28).

Plasmid construction, cell culture and transfection. The fragments of wild-type (WT) and mutated (Mut) 3'-UTRs of CCNE1 and TUSC1 were amplified via PCR from the human cDNA library isolated from human peripheral blood and inserted into the pGL3-promoter vector (Promega Corporation). The PCR thermocycling conditions were as follows: Initial denaturation at $95^{\circ} \mathrm{C}$ for $5 \mathrm{~min}, 35$ cycles of denaturation at $95^{\circ} \mathrm{C}$ for $30 \mathrm{sec}$, annealing at $58^{\circ} \mathrm{C}$ for $30 \mathrm{sec}$, elongation at $72^{\circ} \mathrm{C}$ for $2 \mathrm{~min} \sec$ and final extension at $72^{\circ} \mathrm{C}$ for $5 \mathrm{~min}$. The coding regions of CCNE1 were also generated using PCR amplification and cloned into the expression vector pCMV-Flag (Invitrogen; Thermo Fisher Scientific, Inc.). The human cortical neuronal cell line $\mathrm{HCN}-2$ and the glioma cell lines U251, LN229 and LN18, were cultured for $24 \mathrm{~h}$ in DMEM supplemented with $10 \%$ FBS and $2 \mathrm{mM}$ glutamine (Thermo Fisher Scientific, Inc.). The passaged cells were seeded into 6- or 12-well plates for overnight culture followed by transfection with plasmids $(2 \mu \mathrm{g})$ using Lipofectamine ${ }^{\circledR} 2000$
(5 $\mu$ l) (Invitrogen; Thermo Fisher Scientific, Inc.) for $25 \mathrm{~min}$ at room temperature. The subsequent experiments were performed $48 \mathrm{~h}$ later. In the in vitro functional experiments, the oligonucleotides were chemically synthesized and transfected at a final concentration of $50 \mathrm{nmol} / 1$ according to the manufacturer's instructions (Guangzhou RiboBio Co., Ltd.). Following transfection with oligonucleotides, the cells were cultured in complete DMEM and subsequently harvested for further experiments. All cells were incubated at $37^{\circ} \mathrm{C}$ in an atmosphere of $5 \% \mathrm{CO}_{2}$.

Intracranial glioma model. Nude mice (male BALB/cA-nu; age, 8 weeks) were purchased from the Shanghai Experimental Animal Center (Chinese Academy of Sciences) and maintained under specific pathogen-free conditions. The animals were housed at $22 \pm 2^{\circ} \mathrm{C}$, humidity of $55 \pm 10 \%, 12 / 12$-h light/dark cycle and ad libitum access to water and food. At the start of the experiments, animals weighed $22 \pm 2 \mathrm{~g}$. A total of 15 mice were randomly divided into three groups (Scramble, miR-497 and MIR497HG; $n=5$ each). Luciferase modified-glioma cells were established to overexpress miR-497 or MIR497HG via lentivirus (Shanhai GeneChem Co., Ltd.) and injected intracranially into each mouse at a density of $1 \times 10^{6}$ cells. At 14 days after inoculation, the tumor-bearing mice were infected with luciferin (Shanghai Yeasen Biotechnology Co., Ltd.) and the glioma growth was evaluated via bioluminescence imaging. The mice were sacrificed by cervical dislocation under pentobarbital sodium intraperitoneal anesthesia $(60 \mathrm{mg} / \mathrm{kg})$ to minimize discomfort. The body weight of experimental mice was not significantly different compared with that before tumor cell inoculation. All animal experiments were approved by the Animal Experiment Administration Committee of The Fourth Military Medical University. All experiments were performed in accordance with the recommendations of the Guide for the Care and Use of Laboratory Animals prepared by the National Academy of Sciences and published by the National Institutes of Health (29).

RNA extraction and quantification assay. Total RNA was extracted from glioma cell line U251, LN229 and LN18 using TRIzol $^{\circledR}$ reagent (Invitrogen; Thermo Fisher Scientific, Inc.) according to the manufacturer's instructions. cDNA was generated using TaqMan MicroRNA Reverse Transcription (Thermo Fisher Scientific, Inc.) or PrimerScript RT Reagent kit (Takara Bio, Inc.) according to the manufacturer's instructions. Reverse transcription-quantitative (RT-q)PCR was performed using a CFX96 ${ }^{\mathrm{TM}}$ Real-Time PCR system (Bio-Rad Laboratories, Inc.) with SYBR-Green reagents (cat. no. DRR041A; Takara Bio, Inc.) according to the manufacturer's instructions as previously described (30). The primers were synthesized by Takara Bio, Inc., as follows: MIR497HG forward, 5'-GAG ATCTCTTGTGGGGGTGC-3' and reverse, 5'-ACGTAG CAGGGTGTTTCAGG-3'; CCNE1 forward, 5'-AAGGAG CGGGACACCATGA-3' and reverse, 5'-ACGGTCACGTTT GCCTTCC-3'; TUSC1 forward, 5'-GCCTCTTCCGTCAGG CTTT-3' and reverse, 5'-CGGAGTCGGGTTCCTGTAGA-3' and GAPDH forward, 5'-CTTCAACGACCACTTTGT-3' and reverse, 5'-TGGTCCAGGGGTCTTACT-3'. To analyze miR-497 and miR-588 expression levels, Bulge-Loop ${ }^{\mathrm{TM}}$ miR RT-qPCR primer kit (Guangzhou RiboBio Co., Ltd.) was used 
according to the manufacturer's instructions. The RNA input was normalized to the level of human U6 small nuclear RNA (forward, 5'-GTGCTCGCTTCGGCAGCA-3' and reverse, 5'-CAAAATATGGAACGCTTC-3'). The quantification was performed via the $2^{-\Delta \Delta \mathrm{Cq}}$ method (31).

Western blot analysis. Western blot analysis was performed following harvesting of glioma cell LN229 cells and lysis on ice for 30 min using RIPA buffer supplemented with protease inhibitors (100 mM Tris-HCl, pH 7.4; $150 \mathrm{mM} \mathrm{NaCl;} 5 \mathrm{mM}$ EDTA; $1 \%$ Triton X-100; $1 \%$ deoxycholate acid; 0.1\% SDS, $2 \mathrm{mM}$ phenylmethylsulfonyl fluoride; $1 \mathrm{mM}$ sodium orthovanadate; $2 \mathrm{mM}$ dithiothreitol; $2 \mathrm{mM}$ leupeptin; $2 \mathrm{mM}$ pepstatin). The cell lysate was centrifuged at $10,000 \mathrm{x}$ g for $15 \mathrm{~min}\left(4^{\circ} \mathrm{C}\right)$ and the supernatant was collected to extract the total protein. The concentration levels of the protein samples were determined using the BCA method (Beyotime Institute of Biotechnology) and an $20 \mu \mathrm{l}$ sample was separated via SDS-PAGE (12\%) and transferred onto PVDF membranes. The membranes were blocked at RT with 5\% non-fat dried milk solution for $2 \mathrm{~h}$ and incubated with primary antibodies against CCNE1 (Abcam) and $\beta$-actin $(1: 2,000$; cat. no. EK1002; Boster Biological Technology). Following washing of the membranes three times with PBS-Tween-20 (0.1\%), membranes were incubated with horseradish peroxidase-conjugated secondary antibody (1:5,000; cat. no. BA1056; Boster Biological Technology) for $2 \mathrm{~h}$ at room temperature and visualized with an ECL detection system [Multi Science (Lianke) Biotech Co., Ltd.]. The protein expression was measured using ImageJ software (version ImageJ 2X; National Institutes of Health).

Immunofluorescence staining. The slices of normal, adjacent and glioma tissue derived from patients were examined using in situ hybridization for miR-497 detection (Wuhan Servicebio Technology Co., Ltd.) and counterstained with DAPI. The clinical samples were observed using a laser scanning confocal microscope (FV-1000; Olympus Corporation). Briefly, the tissues were fixed in DEPC for $12 \mathrm{~h}$ at room temperature, followed by dehydration by gradient alcohol, paraffin embedding and sectioning $(8 \mu \mathrm{m})$. The slices were boiled in the retrieval solution for 10-15 min and tissue was marked with liquid blocker pen. Proteinase K (20 $\mu \mathrm{g} / \mathrm{ml}$; Wuhan Servicebio Technology Co., Ltd.) working solution was added to cover objectives and incubated at $37^{\circ} \mathrm{C}$ for $30 \mathrm{~min}$. Sections were washed in pure water, then washed three times in PBS ( $\mathrm{pH}$ 7.4) on a Rocker device for $5 \mathrm{~min}$ each. Then, pre-hybridization solution was added to each section and incubated for $1 \mathrm{~h}$ at $37^{\circ} \mathrm{C}$. After removing the pre-hybridization solution, $\mathrm{miR}-497$ probe hybridization solution $[1 \mu \mathrm{M}$ Carboxyfluorescein (FAM)]-labelled miR-497 probe (5'-ACAAACCACAGTGTGCTGCTG-3') was added overnight at $42^{\circ} \mathrm{C}$. Then, the hybridization solution was removed and the sections were washed in $2 \mathrm{x}$ SSC for $10 \mathrm{~min}$ at $37^{\circ} \mathrm{C}$, in $1 \mathrm{x}$ SSC twice for $5 \mathrm{~min}$ each at $37^{\circ} \mathrm{C}$ and in $0.5 \mathrm{x} \mathrm{SSC}$ for $10 \mathrm{~min}$ at room temperature. The cell nuclei was stained with DAPI for $8 \mathrm{~min}$ in the dark at room temperature. Finally, microscopic examination was performed to take photos under a positive fluorescence microscope. DAPI glows blue by UV excitation wavelength 330-380 $\mathrm{nm}$ and emission wavelength $420 \mathrm{~nm}$; FAM glows green by excitation wavelength 465-495 $\mathrm{nm}$ and emission wavelength 515-555 $\mathrm{nm}$.

Luciferase reporter assay. The target genes of miRNAs were predicted by bioinformatics analysis via TargetScan (32) and miRanda online tools (33). U251 cells ( $1 \times 10^{5}$ cells) were seeded into a 96-well plate and luciferase reporter plasmids (Invitrogen; Thermo Fisher Scientific, Inc.) bearing WT or Mut 3'-UTR of CCNE1 were transfected with miR-497 oligonucleotides (Guangzhou RiboBio Co., Ltd.) and pRL-TK vector by Lipofectamine (Invitrogen; Thermo Fisher Scientific, Inc.). The 3'-UTRs of TUSC1 were transfected with miR-588 oligonucleotides (Guangzhou RiboBio Co., Ltd.) and pRL-TK vector. The cells were harvested and lysed with lysis buffer at $24 \mathrm{~h}$ post-transfection (Promega Corporation). The relative luciferase activity was measured using a Dual Luciferase Reporter Assay system (Promega Corporation) and normalized to the relative activity of Renilla. Each experiment was performed at least five times and the data were analyzed via paired Student's t-test.

Proliferation assay. The proliferation of glioma cells was analyzed via MTT assay. The miR-497- or MIR497HG-overexpressing glioma cells ( $1 \times 10^{5}$ cells) were seeded into 96-well plates and cell proliferation was evaluated at 24, 48, 72 and $96 \mathrm{~h}$ using MTT reagent $(5.0 \mathrm{mg} / \mathrm{ml}$ in PBS). CCNE1 or miR-588 were overexpressed for rescue experiments. Following incubation for $4 \mathrm{~h}$ at $37^{\circ} \mathrm{C}$, the supernatant was removed and the precipitate was dissolved in DMSO (Sigma-Aldrich; Merck KGaA). Spectrophotometric absorbance was measured at $570 \mathrm{~nm}$ using a microplate reader (BioTek Instruments Inc.).

Cell cycle assay. The cell cycle distribution was determined using a BD Accuri ${ }^{\mathrm{TM}}$ C6 Plus Flow Cytometer (BD Biosciences). Briefly, U251 cells were collected and fixed in ice-cold ethanol $\left(70 \%\right.$ in PBS) overnight at $4{ }^{\circ} \mathrm{C}$. The cells were treated with $20 \mathrm{~g} / \mathrm{ml}$ RNase A (Sigma-Aldrich; Merck $\mathrm{KGaA}$ ) for $1 \mathrm{~h}$ at $37^{\circ} \mathrm{C}$ to degrade the RNA, then incubated with $50 \mu \mathrm{g} / \mathrm{ml}$ PI (Sigma-Aldrich; Merck KGaA) in the dark for $30 \mathrm{~min}$ at $4^{\circ} \mathrm{C}$. The DNA content was analyzed via flow cytometry (Accuri ${ }^{\mathrm{TM}}$ C6 Plus, BD Biosciences) and all phases of the cell cycle were analyzed by appropriate gating on the distribution plot and analyzed by FlowJo (Flowjo, LLC; V10).

Statistical analysis. The data were analyzed using SPSS 12.0 software (SPSS, Inc.). Comparisons between groups were performed with an unpaired Student's t-test or one-way ANOVA followed by Student-Newman-Keuls test using GraphPad Prism software (GraphPad Software, Inc.; version 5.0). The correlation was evaluated using Pearson's correlation. Survival analysis was evaluated using the Kaplan-Meier method and assessed using the log-rank test. Data are presented as the mean \pm SEM. The number of independent experimental repeats was described in figure legends. $\mathrm{P}<0.05$ was considered to indicate a statistically significant difference.

\section{Results}

Decreased expression levels of miR-497 and MIR497HG predict poor prognosis of patients with glioma. To investigate 
the expression profile of miR-497 in glioma, in situ hybridization staining was used to analyze the expression levels of this $\mathrm{miR}$ in 27 pairs of glioma and corresponding adjacent paracancerous, as well as normal brain tissue. The results indicated that the number of miR-497-positive cells was significantly decreased in glioma compared with adjacent non-neoplastic and normal brain tissue (Fig. 1A and B). miR-497 is located at the intron of its host gene, MIR497HG (26). The present study verified the expression levels of these miRs in patient tissue samples using RT-qPCR. The data suggested that expression levels of miR-497 and MIR497HG were lower in glioma than in non-neoplastic and normal brain tissue (Fig. 1C and D). In addition, miR-497 expression was highly correlated with that of its host gene MIR497HG (Fig. 1E). Expression levels of miR-497 and MIR497HG decreased as the pathological grade of glioma tumor increased (Fig. 1F and G). Subsequent analysis indicated that higher expression levels of miR-497 and MIR497HG were associated with better prognosis and longer survival time of patients with glioma (Fig. $1 \mathrm{H}$ and I). These data indicated that miR-497 and its host gene MIR497HG were associated with glioma progression and predicted better prognosis of patients with glioma.

Induced overexpression of miR-497 or MIR497HG inhibits proliferation and cell cycle progression in glioma cells. To validate the effects of miR-497 and MIR497HG on glioma, the expression levels of these two targets were determined in glioma (U251, LN229 and LN18) and normal human cortical neuron cell line (HCN-2). The results indicated that the expression levels of miR-497 and MIR497HG were suppressed in the three glioma cell lines compared with HCN-2 cells (Fig. 2A and B). To assess the biological functions of miR-497 and MIR497HG in glioma, these were overexpressed in U251 and LN229 cells. Subsequently, cell proliferation and cell cycle progression were evaluated using MTT and flow cytometry. The data indicated that overexpression of either miR-497 or MIR497HG markedly inhibited proliferation in both cell lines compared with scramble control groups (Fig. 2C and D). Similarly, miR-497 and MIR497HG arrested glioma cells at the $\mathrm{G}_{0} / \mathrm{G}_{1}$ phase (Fig. 2E-H). The luciferase-modified glioma cells were transfected with sequences overexpressing miR-497 or MIR497HG and injected intracranially into nude mice. After 3 weeks, glioma growth was evaluated via bioluminescence imaging and tumor weight measurement, which indicated that miR-497 or MIR497HG overexpression suppressed glioma progression (Fig. 2I-K). These results indicated that the miR-497/MIR497HG axis significantly suppressed glioma cell proliferation both in vitro and in vivo.

CCNE1 is a direct target gene of miR-497 in glioma cells. The in vitro experiments demonstrated that miR-497 and MIR497HG blocked glioma cell cycle progression. To determine the mechanism underlying inhibition of cell proliferation and cell cycle arrest induced by miR-497, bioinformatics analysis (TargetScan and miRanda) was performed to predict the potential targets of miR-497. Although several potential targets that may participate in the proliferation of tumor cells have been identified (34-38), CCNE1 directly regulates the cell cycle of different types of cell and was therefore selected as a putative target of miR-497 (39-42). It was predicted that
miR-497 binds to the 3'-UTR of CCNE1 based on a sequence at 485-492 bp (Fig. 3A). To assess whether miR-497 targets CCNE1 by binding to its 3'-UTR region, U251 cells were co-transfected with WT or Mut 3'-UTR luciferase reporter plasmid and miR-497 mimic or scramble control. Luciferase activity was decreased in cells co-transfected with WT 3'-UTR reporter plasmid and miR-497 mimic, whereas this effect was not noted in cells co-transfected with Mut 3'-UTR reporter plasmid and miR-497 mimic (Fig. 3B). Moreover, overexpression of miR-497 decreased CCNE1 mRNA and protein expression levels (Fig. 3C and D). These data indicated that CCNE1 was a direct target gene of miR-497 in glioma cells.

miR-497 targets CCNE1 inhibition of glioma cell proliferation and CCNE1 predicts poor prognosis of patients with glioma. CCNE1 mRNA expression levels were measured in glioma tissue via RT-qPCR analysis. The results indicated that CCNE1 mRNA expression levels were increased in glioma compared with adjacent non-neoplastic and normal brain tissue (Fig. 3E). Subsequently, the correlation between CCNE1 mRNA and miR-497 expression levels was determined in glioma tissue. The expression levels of CCNE1 mRNA were inversely correlated with miR-497 expression (Fig. 3F). Patients with high CCNE1 expression demonstrated a shorter overall survival period as determined by analysis of samples derived from The Cancer Genome Atlas (TCGA) database and tissue from patients with glioma (Fig. 3G and $\mathrm{H}$ ). In addition, CCNE1 mRNA expression levels were higher in glioma cell lines than in HCN-2 cells (Fig. 3I). Restoration of CCNE1 expression reversed the inhibition of proliferation and induction of cell cycle arrest caused by miR-497 overexpression in U251 cells (Fig. 3J-L). The data suggested that miR-497 targeted CCNE1 inhibition of glioma cell proliferation and CCNE1 predicted poor prognosis of patients with glioma.

MIR497HG regulates glioma cell proliferation via the miR-588/TUSC1 axis. Bioinformatics analysis was used to identify target miRs of MIR497HG. miR-588 was predicted to interact with MIR497HG and this was verified by the suppression of miR-588 levels in MIR497HG-overexpressing glioma cells (Fig. 4A and B). The data further indicated that miR-588 specifically bound to the 3'-UTR of TUSC1 via complementary interactions, suggesting that TUSC1 expression could be suppressed by miR-588 (Fig. 4C). This finding was confirmed via RT-qPCR analysis, which indicated significantly decreased TUSC1 mRNA expression in miR-588-overexpressing glioma cells (Fig. 4D). In addition, luciferase reporter assay indicated that miR-588 decreased TUSC1 3'-UTR activity in glioma cells (Fig. 4E). The expression levels of miR-588 increased, while those of TUSC1 decreased in glioma compared with paracancerous and normal tissue (Fig. 4F and G).

The expression levels of miR-588 exhibited an inverse correlation with those of MIR497HG in glioma tissue (Fig. 4H). High levels of miR-588 predicted poor patient prognosis, whereas high levels of TUSC1 indicated longer survival time of patients with primary glioma (Fig. 4I and J). TCGA database indicated decreased expression levels of TUSC1 in glioma compared with non-tumor tissue. However, the expression levels of TUSC1 were not associated with the prognosis of patients with glioblastoma (GBM), which did 
A
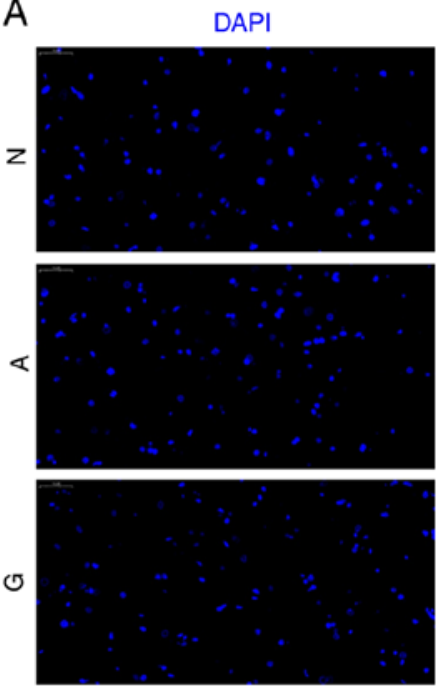

B

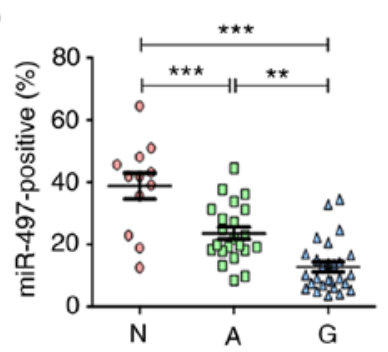

E

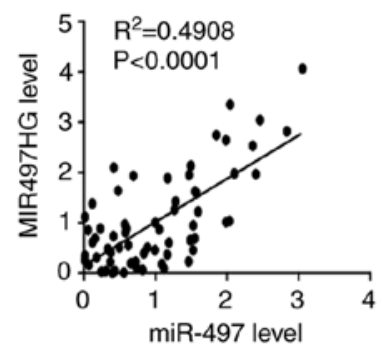

$\mathrm{H}$

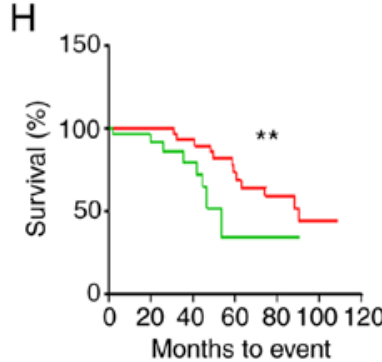

miR-497
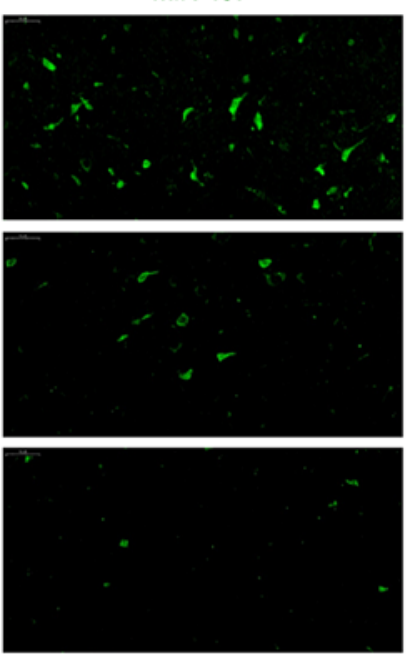

C

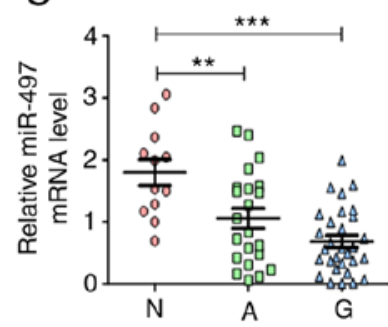

F

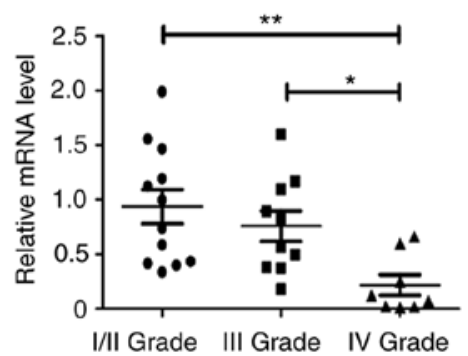

DAPI/miR-497
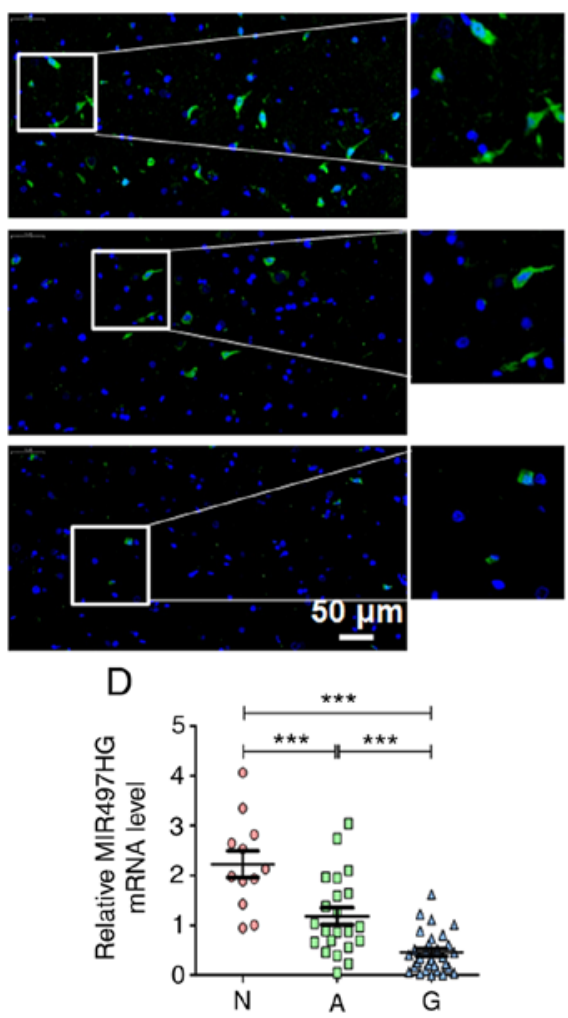

G
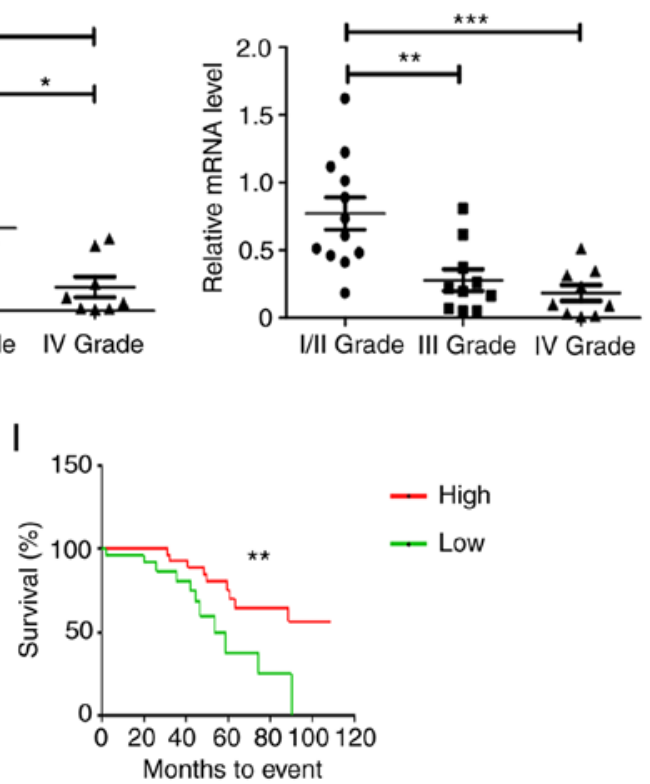

Figure 1. Decreased expression levels of miR-497/MIR497HG predict poor prognosis of patients with glioma. (A) Fluorescent in situ hybridization assay was performed to evaluate the expression levels of miR-497 in N (n=12), A ( $n=22)$ and $\mathrm{G}(\mathrm{n}=30)$. The magnification of the enlarged area is $\mathrm{x} 40$. (B) Positive miR-497 staining in clinical tissue samples was measured and quantified. mRNA expression levels of (C) miR-497 and (D) MIR497HG were detected in tissue using reverse transcription-quantitative PCR analysis. (E) Correlation between miR-497 and MIR497HG expression levels in tissue $\left(\mathrm{R}^{2}=0.4908\right.$; $\left.\mathrm{n}=64\right)$. Expression levels of (F) miR-497 and (G) MIR497HG in tissue with different pathological grades. Correlation between expression levels of (H) miR497 or (I) MIR497HG and the survival time of patients $(\mathrm{n}=60)$. Data are presented as the mean \pm SEM. ${ }^{*} \mathrm{P}<0.05,{ }^{* *} \mathrm{P}<0.01,{ }^{* * * *} \mathrm{P}<0.001$. miR, microRNA; MIR497HG, miR-497 host gene; N, normal; A, adjacent; G, glioma.

not distinguish between patients with primary and recurrent glioma (Fig. S1).

U251 cells were transfected with MIR497HG or MIR497HG + miR-588-expressing plasmids to assess whether
TUSC1 was a downstream target of MIR497HG and miR-588. Elevated MIR497HG expression induced upregulation of TUSC1 mRNA expression and inhibited cell proliferation, while overexpression of miR-588 abrogated these effects (Fig. 4K and L). These 
A
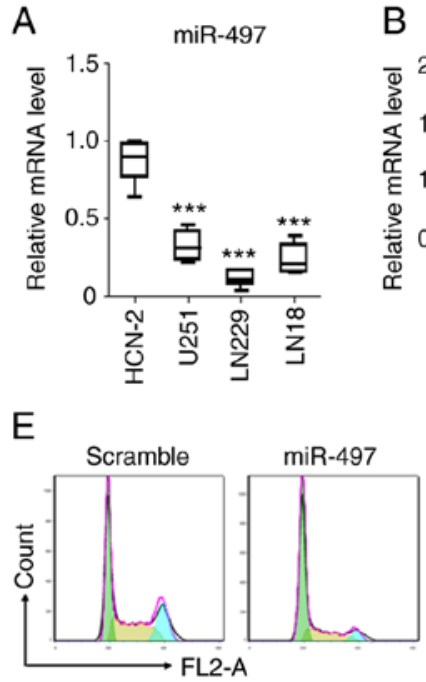

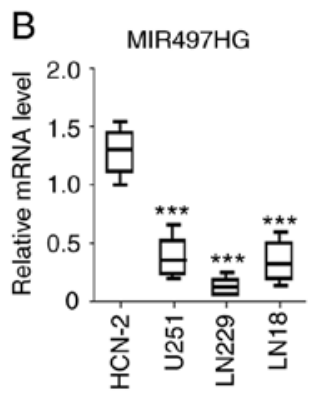

$\mathrm{F}$

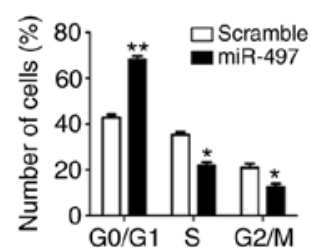

C $\quad-$ U251 scramble

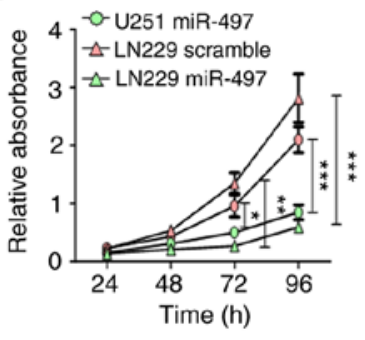

G

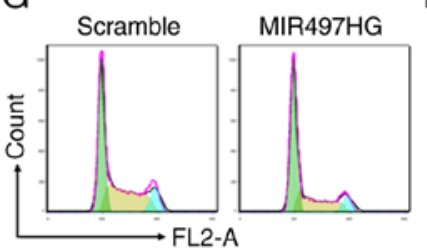

D $\quad-$ U251 vehicle

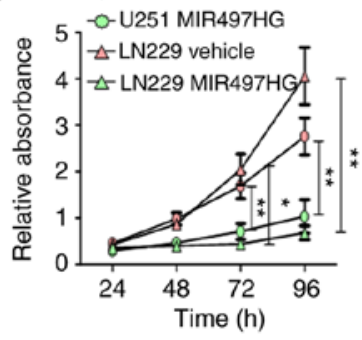

$\mathrm{H}$

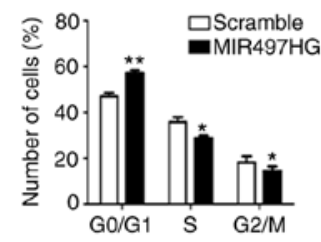

।

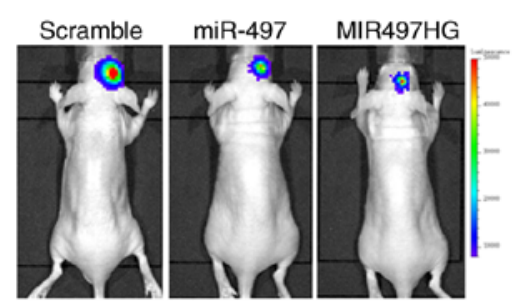

$J$

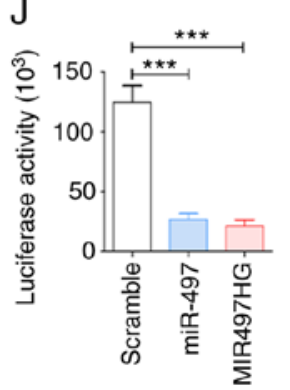

$\mathrm{K}$

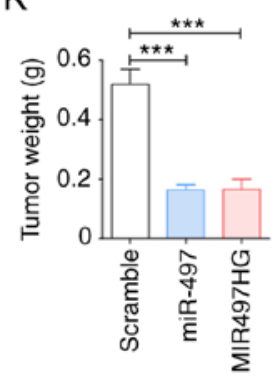

Figure 2. miR-497 and MIR497HG suppress glioma cell proliferation and cell cycle arrest. Expression levels of (A) miR-497 and (B) MIR497HG were determined in glioma and normal human cortical neuron cell lines (HCN-2) via reverse transcription-quantitative PCR analysis. MTT assay was performed to evaluate the proliferation of glioma cells transfected with (C) miR-497 or (D) MIR497HG plasmids. (E and F) miR-497 overexpression was induced in glioma cells and the cell cycle was examined via flow cytometry. (G and H) MIR497HG overexpression was induced in glioma cells and the cell cycle was examined via flow cytometry. (I and J) Luciferase-modified glioma cells overexpressing miR-497 or MIR497HG were injected intracranially into nude mice. Glioma development was evaluated using bioluminescence imaging following 3 weeks of tumor growth. (K) Tumors were excised, and weight was measured following animal sacrifice. Data are presented as the mean \pm SEM $(n=5) .{ }^{*} \mathrm{P}<0.05,{ }^{* *} \mathrm{P}<0.01,{ }^{* * *} \mathrm{P}<0.001$ vs. control. miR, microRNA; MIR497HG, miR-497 host gene.

results demonstrated a novel signaling pathway that regulates glioma cell proliferation via the MIR497HG/miR-588/TUSC1 axis.

\section{Discussion}

Accumulating evidence has indicated that abnormal miR-497 expression contributes to carcinogenesis and progression of multiple types of cancer, including glioma (43-45). In the present study, expression levels of miR-497 and its host gene MIR497HG were significantly downregulated in human glioma tissue, as well as in glioma cell lines. The expression levels of miR-497 or MIR497HG were inversely correlated with tumor grade. Lower miR-497 and MIR497HG expression levels were correlated with poor prognosis of patients with glioma. These results indicated that miR-497 and MIR497HG expression levels correlated with pathological features and prognosis of these patients. It has been previously reported that the lncRNA MIR497HG may be the actual host gene of miR-497 (26). However, the present study indicated that miR-497 and lncRNA MIR497HG shared similar expression profiles in both clinical glioma tissue and glioma cell lines. In addition, histone $\mathrm{H} 3 \mathrm{~K} 27$ me 3 modification is the hallmark event of promoter methylation. Chromatin immunoprecipitation-sequencing data derived from glioma cells from the Cistrome Project (cistrome.org/) exhibit only one unique peak corresponding to a strong signal of $\mathrm{H} 3 \mathrm{~K} 4 \mathrm{me} 3$ near the region of MIR497HG and miR-497 (Gene Expression Omnibus ID: GSM2288202; CistromeDB ID: 82969) (46). This indicates that MIR497HG and miR-497 are simultaneously transcribed in glioma cells. Considering that the histone modifications include heterogeneity and plasticity, the transcriptional levels of MIR497HG and miR-497 may be different in different types of cell or tissue.

The present study is not the first to analyze miR-497 expression and its correlation with patient prognosis. Lu et al (45) demonstrated that miR-497 expression levels are downregulated in glioma tissue and associated with poor disease progression and decreased overall survival, suggesting a tumor suppressor function and a potential therapeutic application of miR-497 in glioma. Regazzo et al (47) indicated that patients with GBM, which is considered the most common and malignant subtype of glioma, are more likely to exhibit downregulated expression levels of miR-497 compared with patients those with lower tumor grades. This suggests that serum miR-497 levels may be a novel diagnostic marker for clinical application in patients with glioma (47). Feng et al (48) revealed that miR-497 expression is suppressed in human glioma tissue and associated with 


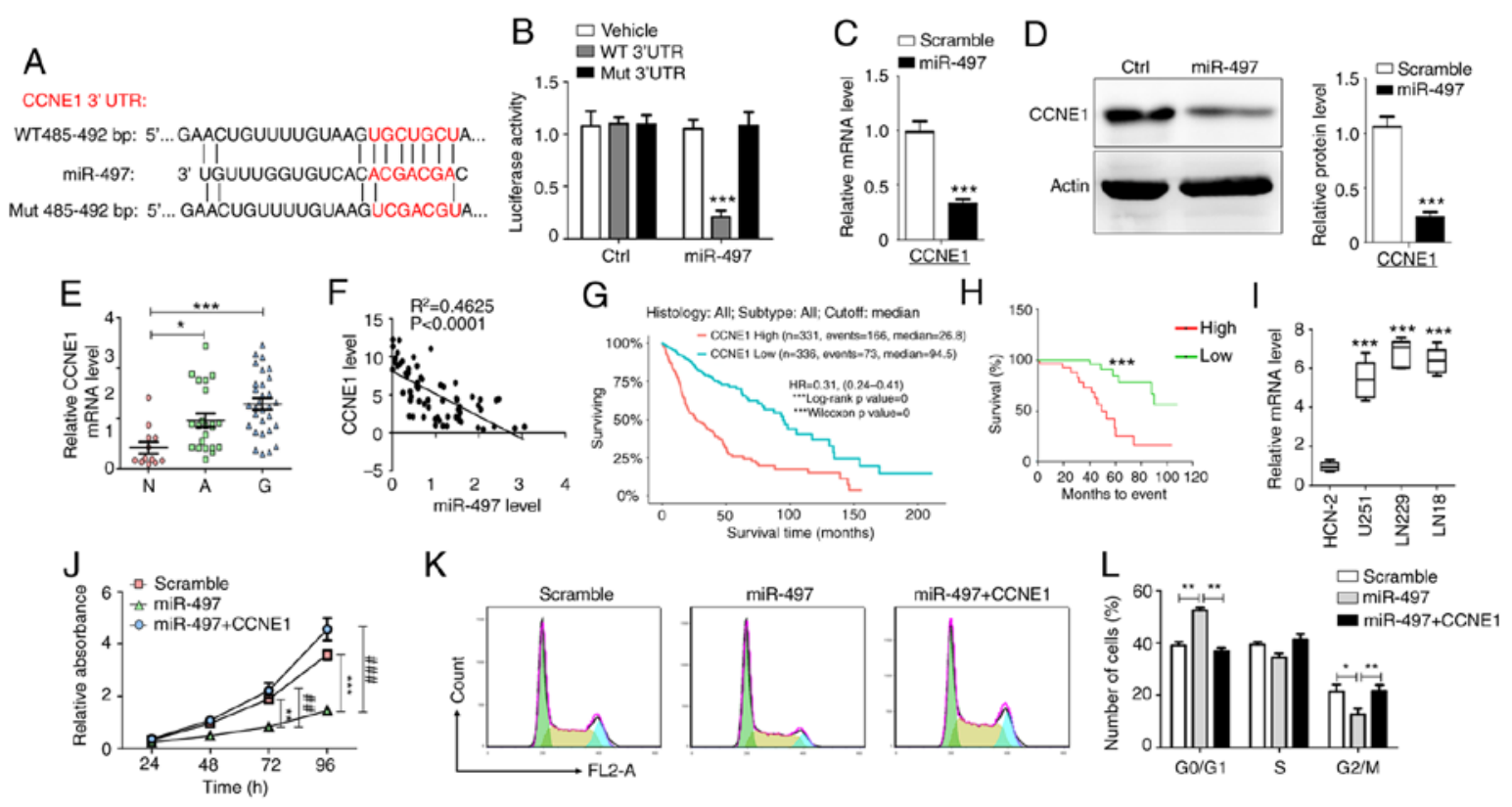

Figure 3. CCNE1 is a direct functional downstream target of miR-497. (A) WT and Mut binding sites of miR-497 in the 3'-UTR of CCNE1. (B) Relative luciferase activity was determined in U251 cells following co-transfection of miR-497 and luciferase reporter plasmid with WT or Mut CCNE1 3'-UTR (n=5). Glioma cells were transfected with miR-497 and (C) mRNA and (D) protein levels of CCNE1 were detected (n=5). (E) mRNA levels of CCNE1 were determined using RT-qPCR in N $(n=12)$, A $(n=22)$ and $G(n=30)$. (F) Correlation between CCNE1 and miR-497 $\left(R^{2}=0.4625\right)$. $(\mathrm{G})$ Expression levels of CCNE1 correlated with overall survival time of 667 patients derived from The Cancer Genome Atlas database. $(\mathrm{H})$ Correlation between expression levels of CCNE1 and the survival time of patients $(\mathrm{n}=60)$. (I) RT-qPCR analysis was performed to detect CCNE1 mRNA expression in glioma and HCN-2 cells (n=5). (J) MTT assay demonstrated the effects of CCNE1 overexpression on inhibition of cell proliferation induced by miR-497 ( $\mathrm{n}=6)$. (K and L) Flow cytometry analysis indicated the effects of CCNE1 overexpression on cell cycle arrest induced by miR-497 $(\mathrm{n}=6)$. Data are presented as the mean $\pm \mathrm{SEM}$. ${ }^{*} \mathrm{P}<0.05,{ }^{* * *}$ or ${ }^{\# \# P} \mathrm{P}<0.01,{ }^{* * * *}$ or ${ }^{\# \# \#} \mathrm{P}<0.001$. CCNE1, cyclin E1; miR, microRNA; WT, wild-type; Mut, mutant; UTR, untranslated region; RT-q, reverse transcription-quantitative; N, normal; A, adjacent; G, glioma; Ctrl, control.

B
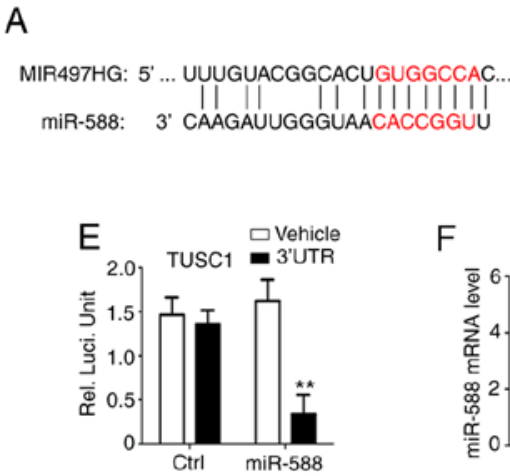

I

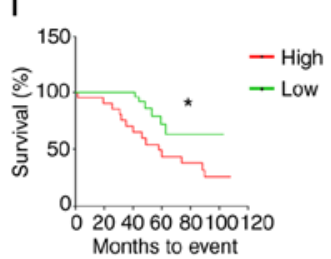

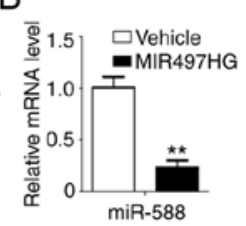

$\mathrm{F}$

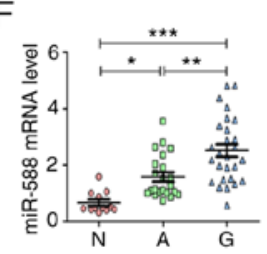

$J$

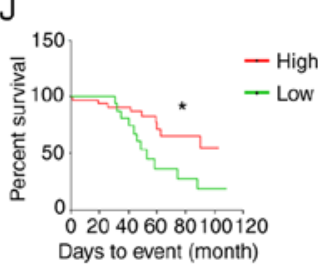

C

TUSC1 3' UTR:

WT 478-485 bp: 5'... GCUAUUCUUUCAUAUGUGGCCAA... miR-588: 3' CAAGAUUGGGUA--ACACCGGUU

G

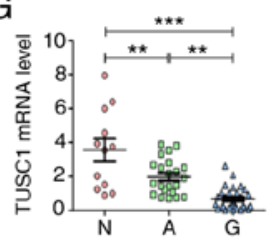

$\mathrm{K}$

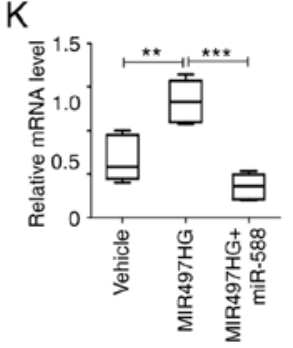

D

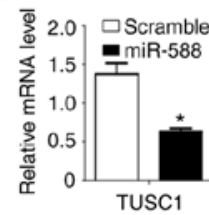

$\mathrm{H}$

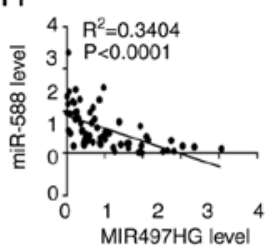

$\mathrm{L}$

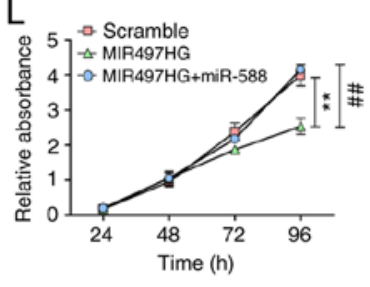

Figure 4. MIR497HG regulates cell proliferation and cell cycle via the miR-588/TUSC1 signaling axis. (A) Bioinformatic analysis indicated that miR-588 may be a specific downstream miRNA of MIR497HG. (B) Glioma cells overexpressing MIR497HG were established and mRNA levels of miR-588 were detected $(\mathrm{n}=5)$. (C) Bioinformatics analysis indicated that TUSC1 may be a specific downstream target of miR-588. (D) Glioma cells overexpressing miR-588 were established and mRNA expression levels of TUSC1 were detected (n=5). (E) Relative luciferase activity was determined in U251 cells following co-transfection of miR-588 plasmid with luciferase reporter plasmid containing TUSC1 3'-UTR. mRNA expression levels of (F) miR-588 and (G) TUSC1 were detected in N $(\mathrm{n}=12), A(\mathrm{n}=22)$ and $\mathrm{G}(\mathrm{n}=30)$ using reverse transcription-quantitative PCR analysis. (H) Correlation between miR-588 and MIR497HG. Correlation between the expression levels of (I) miR-588 or (J) TUSC1 and survival time of patients $(\mathrm{n}=60)$ was analyzed. $(\mathrm{K})$ Effect of miR-588 was evaluated on the increase in TUSC1 expression levels induced by MIR497HG. (L) MTT assay indicated that miR-588 abrogated the inhibition of cell proliferation induced by MIR497HG. Data are presented as the mean \pm SEM. ${ }^{*} \mathrm{P}<0.05,{ }^{* *}$ or ${ }^{\# /} \mathrm{P}<0.01,{ }^{* * * *} \mathrm{P}<0.001$. MIR497HG, miR-497 host gene; miR, microRNA; TUSC1, tumor suppressor candidate 1; UTR, untranslated region; RT-qPCR, reverse transcription-quantitative; N, normal; A, adjacent; G, glioma; Ctrl, control. 


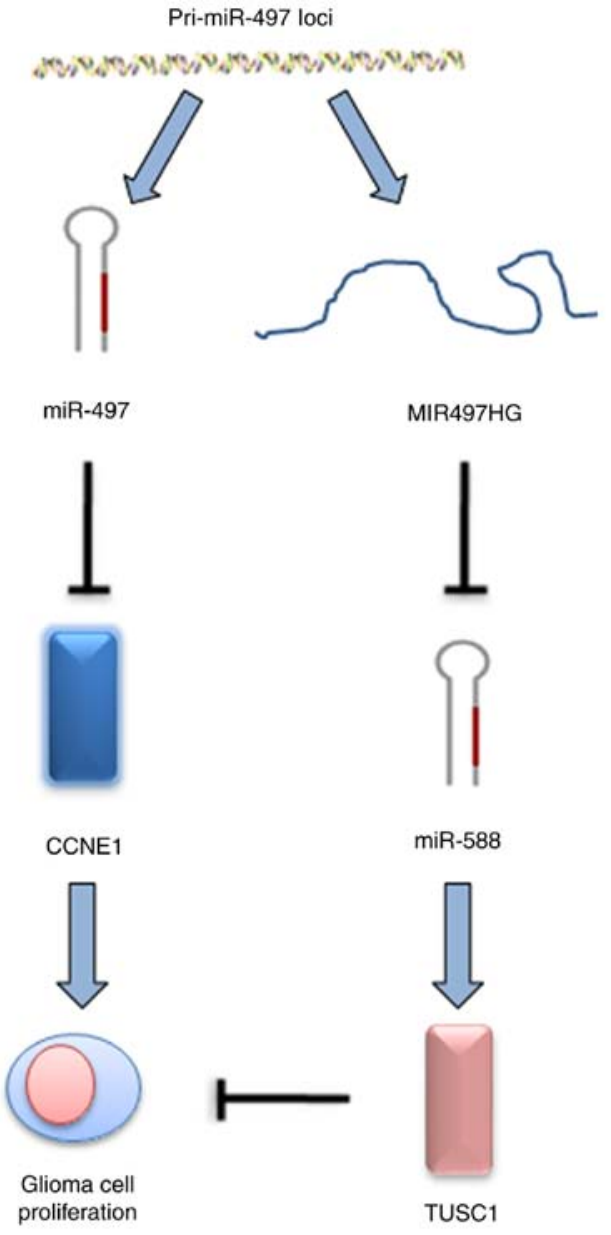

Figure 5. Schematic diagram demonstrating the modulation of cell proliferation caused by miR-497/CCNE1 and MIR497HG/miR-588/TUSC1 signaling pathways in glioma. miR, microRNA; CCNE1, cyclin E1; MIR497HG, miR-497 host gene; TUSC1, tumor suppressor candidate 1; pri, primary.

higher degree of angiogenesis and poor prognosis. In addition, a genome-wide miRNA sequence survey was performed and Yang et al (49) reported that the expression levels of miR-497 are significantly decreased in serum samples of patients with astrocytoma compared with those of normal control subjects. The present study verified the aforementioned findings, suggesting that miR-497 and MIR497HG are tumor suppressors and can be used as prognostic biomarkers in glioma. The findings of the present study suggested that the combination of miR-497 and its host gene MIR497HG can target glioma progression. However, contradictory findings have also been reported $(50,51)$. Based on an online dataset analysis, Lan et al (50) demonstrated that miR-497 is upregulated in glioma tissue and that hypoxia induces expression levels of miR-497 and its transcriptional levels by binding to the hypoxia response element and promoting chemoresistance in glioma cells via programmed cell death. Zhu et al (51) revealed that miR-497 expression levels are upregulated in glioma cell lines and that this conferred resistance to temozolomide in these cells by targeting the $\mathrm{mTOR} / \mathrm{Bcl}-2$ pathway. However, the aforementioned study did not investigate the expression profile of miR-497 in glioma tissue samples (51). The exact causes of the different expressions of miR-497 in glioma remain unclear and require further investigation. Therefore, uniform and globally standardized experimental protocols have to be developed in specimen collection, storage, $\mathrm{miR}$ extraction, purification, profiling and analytical methods. Additional multi-center studies and cohorts are required to achieve consistent and robust results that can be applied for clinical use.

In cancer progression, miRs function as regulatory molecules and downregulate the expression levels of downstream target genes, which are involved in cell differentiation, proliferation, angiogenesis and other biological processes (52). Here, induced overexpression of miR-497 or MIR497HG inhibited cell proliferation and cell cycle arrest in vitro. CCNE1 is an important cell cycle regulator that is required for $\mathrm{G}_{1} / \mathrm{S}$ transition (53). Bioinformatics analysis and luciferase assay indicated that CCNE1 was a direct target that was suppressed by miR-497. Rescue experiments indicated that overexpression of CCNE1 abrogated inhibition of cell proliferation and cell cycle arrest caused by miR-497, which was consistent with a previous study (54). The expression levels and function of miR-588 have been investigated in gastric (55) and liver cancer (56); this $\mathrm{miR}$ functions either as a tumor suppressor or as an oncogenic molecule $(54,57-59)$. TUSC1 is a putative tumor suppressor gene that has been shown to serve a role in different types of cancer, including GBM $(60,61)$. In the present study, miR-588/TUSC1 was identified as the downstream signaling axis of MIR497HG, which participated in the regulation of glioma cell proliferation and cell cycle progression. High levels of TUSC1 indicated longer survival time of patients with primary glioma. However, the TCGA database indicated that TUSC1 expression was not associated with the prognosis of patients with glioblastoma. Considering that the TCGA database did not distinguish between patients with primary and recurrent glioma, this inconsistent conclusion may be explained due to the differences between primary and recurrent gliomas. To the best of our knowledge, the present study is the first to demonstrate the importance of the MIR497HG/miR-588/TUSC1 signaling pathway in glioma progression (Fig. 5).

In conclusion, the present study indicated that miR-497 and MIR497HG expression levels were downregulated in glioma tissue and associated with poor disease prognosis. Overexpression of miR-497 or MIR497HG inhibited proliferation and cell cycle progression in glioma cells via downregulation of CCNE1 expression and inhibition of the miR-588/TUSC1 signaling pathway. The present study may provide new insight into the molecular mechanism of glioma progression and aid the development of potential therapeutic targets and prognostic biomarkers for this cancer.

\section{Acknowledgements}

The authors would like to thank Dr Shuang Wu, Fourth Military Medical University for providing the space and equipment for experiments.

\section{Funding}

The present study was supported by grants from National Natural Science Foundation of China (grant no. 81502145) and National Natural Science Foundation of Shaanxi (grant no. 2016JQ8017). 


\section{Availability of data and materials}

The datasets used and/or analyzed during the current study are available from the corresponding author on reasonable request.

\section{Authors' contributions}

LYJ and MW performed the experiments. YYL performed data analysis. ZLD and SZL designed and directed the study and wrote the manuscript. MW and YYL confirm the authenticity of all the raw data. All authors have read and approved the final version of the manuscript.

\section{Ethics approval and consent to participate}

The present study was approved by the Ethics Committee of Xijing Hospital of Fourth Military Medical University (approval no. 20190238) and was performed in accordance with the Declaration of Helsinki. Written informed consent was obtained from all patients.

\section{Patient consent for publication}

Not applicable.

\section{Competing interests}

The authors declare that they have no competing interests.

\section{References}

1. Siegel RL, Miller KD and Jemal A: Cancer statistics, 2019. CA Cancer J Clin 69: 7-34, 2019.

2. Diamandis $P$ and Aldape KD: Insights from molecular profiling of adult glioma. J Clin Oncol 35: 2386-2393, 2017.

3. Laug D, Glasgow SM and Deneen B: A glial blueprint for gliomagenesis. Nat Rev Neurosci 19: 393-403, 2018.

4. He L and Hannon GJ: MicroRNAs: Small RNAs with a big role in gene regulation. Nat Rev Genet 5: 522-531, 2004.

5. Zhao J,Li H, Zhao S, Wang E, Zhu J, Feng D, Zhu Y, Dou W, Fan Q, $\mathrm{Hu}$ J, et al: Epigenetic silencing of miR-144/451a cluster contributes to HCC progression via paracrine HGF/MIF-mediated TAM remodeling. Mol Cancer 20: 46, 2021.

6. Li SZ, Ren KX, Zhao J, Wu S, Li J, Zang J, Fei Z and Zhao JL: miR-139/PDE2A-Notch1 feedback circuit represses stemness of gliomas by inhibiting Wnt/ $/$-catenin signaling. Int J Biol Sci 17: 3508-3521, 2021.

7. Wu Q, Luo X, Terp MG, Li Q, Li Y, Shen L, Chen Y, Jacobsen K, Bivona TG, Chen H, et al: DDX56 modulates post-transcriptional Wnt signaling through miRNAs and is associated with early recurrence in squamous cell lung carcinoma. Mol Cancer 20: 108, 2021

8. Godlewski J, Krichevsky AM, Johnson MD, Chiocca EA and Bronisz A: Belonging to a network-microRNAs, extracellular vesicles, and the glioblastoma microenvironment. Neuro Oncol 17: 652-662, 2015.

9. Chen X, Liang H, Zhang J, Zen K and Zhang CY: Secreted microRNAs: A new form of intercellular communication. Trends Cell Biol 22: 125-132, 2012.

10. Hassan A, Mosley J, Singh S and Zinn PO: A comprehensive review of genomics and noncoding RNA in gliomas. Top Magn Reson Imaging 26: 3-14, 2017.

11. Peng Z, Zhu Y, Zhang Y, Wilhelmsen K, Jia C, Jin J, Xue Q, Feng $\mathrm{X}$, Zhang $\mathrm{F}$ and $\mathrm{Yu} \mathrm{B}$ : Effects of ghrelin on pulmonary NOD2 mRNA expression and NF- $\kappa \mathrm{B}$ activation when protects against acute lung injury in rats challenged with cecal ligation and puncture. Int Immunopharmacol 13: 440-445, 2012.

12. Fan Q, Zhao P, Li J, Xie X, Xu M, Zhang Y, Mu D, Li W, Sun R, Liu W, et al: $17 \beta$-Estradiol administration attenuates seawater aspiration-induced acute lung injury in rats. Pulm Pharmacol Ther 24: 673-681, 2011.
13. Qiu YB, Wan BB, Liu G, Wu YX, Chen D, Lu MD, Chen JL, Yu RQ, Chen DZ and Pang QF: Nrf2 protects against seawater drowning-induced acute lung injury via inhibiting ferroptosis. Respir Res 21: 232, 2020.

14. Buntwal L, Sassi M, Morgan AH, Andrews ZB and Davies JS: Ghrelin-mediated hippocampal neurogenesis: Implications for health and disease. Trends Endocrinol Metab 30: 844-859, 2019.

15. Menigatti M, Staiano T, Manser CN, Bauerfeind $P$, Komljenovic A, Robinson M, Jiricny J, Buffoli F and Marra G: Epigenetic silencing of monoallelically methylated miRNA loci in precancerous colorectal lesions. Oncogenesis 2: e56, 2013.

16. Xie Y, Wei RR, Huang GL, Zhang MY, Yuan YF and Wang HY: Checkpoint kinase 1 is negatively regulated by miR-497 in hepatocellular carcinoma. Med Oncolo 31: 844, 2014.

17. Yang J, Ye Z, Mei D, Gu H and Zhang J: Long noncoding RNA DLX6-AS1 promotes tumorigenesis by modulating miR-497-5p/FZD4/FZD6/Wnt//3-catenin pathway in pancreatic cancer. Cancer Manag Res 11: 4209-4221, 2019.

18. Özata DM, Caramuta S, Velázquez-Fernández D, Akçakaya P, Xie H, Höög A, Zedenius J, Bäckdahl M, Larsson C and Lui WO: The role of microRNA deregulation in the pathogenesis of adrenocortical carcinoma. Endocr Relat Cancer 18: 643-655, 2011.

19. Wei Z, Hu X, Liu J, Zhu W, Zhan X and Sun S: MicroRNA-497 upregulation inhibits cell invasion and metastasis in T24 and BIU-87 bladder cancer cells. Mol Med Rep 16: 2055-2060, 2017.

20. Sen LS, Karakoyun B, Yeğen C, Akkiprik M, Yüksel M, Ercan F, Özer A and Yeğen BÇ: Treatment with either obestatin or ghrelin attenuates mesenteric ischemia-reperfusion-induced oxidative injury of the ileum and the remote organ lung. Peptides 71: 8-19, 2015.

21. Luo M, Shen D, Zhou X, Chen X and Wang W: MicroRNA-497 is a potential prognostic marker in human cervical cancer and functions as a tumor suppressor by targeting the insulin-like growth factor 1 receptor. Surgery 153: 836-847, 2013.

22. Maura F, Cutrona G, Mosca L, Matis S, Lionetti M, Fabris S, Agnelli L, Colombo M, Massucco C, Ferracin M, et al: Association between gene and miRNA expression profiles and stereotyped subset \#4 B-cell receptor in chronic lymphocytic leukemia. Leuk Lymphoma 56: 3150-3158, 2015.

23. Troppan K, Wenzl K, Pichler M, Pursche B, Schwarzenbacher D, Feichtinger J, Thallinger GG, Beham-Schmid C, Neumeister P and Deutsch A: miR-199a and miR-497 are associated with better overall survival due to increased chemosensitivity in diffuse large B-cell lymphoma patients. Int J Mol Sci 16: 18077-18095, 2015.

24. Matthay MA, McAuley DF and Ware LB: Clinical trials in acute respiratory distress syndrome: Challenges and opportunities. Lancet Respir Med 5: 524-534, 2017.

25. Yang J, Yang FJ, Wang YG, Su GF and Miao X: LncRNA MIR497HG inhibits proliferation and migration of retinal endothelial cells under high-level glucose treatment via miRNA-128-3p/SIRT1 axis. Eur Rev Med Pharmacol Sci 24: 5871-5877, 2020

26. Zhuang C, Liu Y, Fu S, Yuan C, Luo J, Huang X, Yang W, Xie W and Zhuang C: Silencing of lncRNA MIR497HG via CRISPR/Cas13d induces bladder cancer progression through promoting the crosstalk between Hippo/Yap and TGF- $\beta / \mathrm{Smad}$ signaling. Front Mol Biosci 7: 616768, 2020.

27. Louis DN, Ohgaki H, Wiestler OD, Cavenee WK, Burger PC, Jouvet A, Scheithauer BW and Kleihues P: The 2007 WHO classification of tumours of the central nervous system. Acta Neuropathol 114: 97-109, 2007.

28. Ganini C, Amelio I, Bertolo R, Bove P, Buonomo OC, Candi E, Cipriani C, Di Daniele N, Juhl H, Mauriello A, et al: Global mapping of cancers: The cancer genome atlas and beyond. Mol Oncol: Jul 10, 2021 (Epub ahead of print).

29. Bayne K: Revised guide for the care and use of laboratory animals available. American Physiological Society. Physiologist 39: 199, 208-211, 1996.

30. Wang H, Yan X, Ji LY, Ji XT, Wang P, Guo SW and Li SZ: miR-139 Functions as an antioncomir to repress glioma progression through targeting IGF-1 R, AMY-1, and PGC-1 $\beta$. Technol Cancer Res Treat 16: 497-511, 2017.

31. Livak KJ and Schmittgen TD: Analysis of relative gene expression data using real-time quantitative PCR and the 2(-Delta Delta C(T)) method. Methods 25: 402-408, 2001.

32. Peterson SM, Thompson JA, Ufkin ML, Sathyanarayana $P$, Liaw L and Congdon CB: Common features of microRNA target prediction tools. Front Genet 5: 23, 2014. 
33. Riffo-Campos AL, Riquelme I and Brebi-Mieville P: Tools for sequence-based miRNA Target Prediction: What to Choose? Int J Mol Sci 17: 1987, 2016.

34. Chae DK, Park J, Cho M, Ban E, Jang M, Yoo YS, Kim EE, Baik JH and Song EJ: MiR-195 and miR-497 suppress tumorigenesis in lung cancer by inhibiting SMURF2-induced TGF- $\beta$ receptor I ubiquitination. Mol Oncol 13: 2663-2678, 2019.

35. Feng L, Cheng K, Zang R, Wang Q and Wang J: miR-497-5p inhibits gastric cancer cell proliferation and growth through targeting PDK3. Biosci Rep 39: BSR20190654, 2019.

36. Hassan N, Zhao JT, Glover A, Robinson BG and Sidhu SB: Reciprocal interplay of miR-497 and MALAT1 promotes tumourigenesis of adrenocortical cancer. Endocr Relat Cancer 26: 677-688, 2019.

37. Xia Y, Hu C, Lian L, Hui K, Wang L, Qiao Y, Liu L, Liang L and Jiang X: miR497 suppresses malignant phenotype in nonsmall cell lung cancer via targeting KDR. Oncol Rep 42: 443-452, 2019.

38. Yang L, Cai Y, Zhang D, Sun J, Xu C, Zhao W, Jiang W and Pan C: miR-195/miR-497 Regulate CD274 expression of immune regulatory ligands in triple-negative breast cancer. J Breast Cancer 21: 371-381, 2018.

39. Zhang Y, Li X, Zhang J and Mao L: E6 hijacks KDM5C/lnc_000231/miR-497-5p/CCNE1 axis to promote cervical cancer progression. J Cell Mol Med 24: 11422-11433, 2020 .

40. Han Z, Zhang Y, Yang Q, Liu B, Wu J, Zhang Y, Yang C and Jiang Y: miR-497 and miR-34a retard lung cancer growth by co-inhibiting cyclin E1 (CCNE1). Oncotarget 6: 13149-13163, 2015.

41. Wei W, Zhang WY, Bai JB, Zhang HX, Zhao YY, Li XY and Zhao SH: The NF- $\mathrm{BB}$-modulated microRNAs miR-195 and miR-497 inhibit myoblast proliferation by targeting Igflr, Insr and cyclin genes. J Cell Sci 129: 39-50, 2016

42. Furuta M,Kozaki K, Tanimoto K, Tanaka S, Arii S, Shimamura T, Niida A, Miyano S and Inazawa J: The tumor-suppressive miR-497-195 cluster targets multiple cell-cycle regulators in hepatocellular carcinoma. PLoS One 8: e60155, 2013.

43. Luo G, He K, Xia Z, Liu S, Liu H and Xiang G: Regulation of microRNA-497 expression in human cancer. Oncol Lett 21: 23, 2021.

44. Boldrin E, Gaffo E, Niedermayer A, Boer JM, Zimmermann M, Weichenhan D, Claus R, Münch V, Sun Q, Enzenmüller S, et al: MicroRNA-497/195 is tumor-suppressive and cooperates with CDKN2A/B in pediatric acute lymphoblastic leukemia. Blood: Jun 7, 2021 (Epub ahead of print)

45. Lu F, Ye Y, Zhang H, He X, Sun X, Yao C, Mao H, He X, Qian C, Wang B, et al: miR-497/Wnt3a/c-jun feedback loop regulates growth and epithelial-to-mesenchymal transition phenotype in glioma cells. Int J Biol Macromol 120: 985-991, 2018

46. Turcan S, Makarov V, Taranda J, Wang Y, Fabius AWM, Wu W, Zheng Y, El-Amine N, Haddock S, Nanjangud G, et al: Mutant-IDH1-dependent chromatin state reprogramming, reversibility, and persistence. Nat Genet 50: 62-72, 2018.

47. Regazzo G, Terrenato I, Spagnuolo M, Carosi M, Cognetti G Cicchillitti L, Sperati F, Villani V, Carapella C, Piaggio G, et al: A restricted signature of serum miRNAs distinguishes glioblastoma from lower grade gliomas. J Exp Clin Cancer Res 35: 124, 2016.
48. Feng F, Kuai D, Wang H, Li T, Miao W, Liu Y and Fan Y: Reduced expression of microRNA-497 is associated with greater angiogenesis and poor prognosis in human gliomas. Hum Pathol 58: 47-53, 2016

49. Yang C, Wang C, Chen X, Chen S, Zhang Y, Zhi F, Wang J, Li L, Zhou X, Li N, et al: Identification of seven serum microRNAs from a genome-wide serum microRNA expression profile as potential noninvasive biomarkers for malignant astrocytomas. Int J Cancer 132: 116-127, 2013.

50. Lan J, Xue Y, Chen H, Zhao S, Wu Z, Fang J, Han C and Lou M: Hypoxia-induced miR-497 decreases glioma cell sensitivity to TMZ by inhibiting apoptosis. FEBS Lett 588: 3333-3339, 2014.

51. Zhu D, Tu M, Zeng B, Cai L, Zheng W, Su Z and Yu Z: Up-regulation of miR-497 confers resistance to temozolomide in human glioma cells by targeting $\mathrm{mTOR} / \mathrm{Bcl}-2$. Cancer Med 6: 452-462, 2017.

52. Shenouda SK and Alahari SK: MicroRNA function in cancer: Oncogene or a tumor suppressor? Cancer Metastasis Rev 28: 369-378, 2009

53. Yang R, Xing L, Zheng X, Sun Y, Wang X and Chen J: The circRNA circAGFG1 acts as a sponge of miR-195-5p to promote triple-negative breast cancer progression through regulating CCNE1 expression. Mol Cancer 18: 4, 2019.

54. Shen L, Orillion A and Pili R: Histone deacetylase inhibitors as immunomodulators in cancer therapeutics. Epigenomics 8: 415-428, 2016.

55. Chen Y, Zhang J, Gong W, Dai W, Xu X and Xu S: miR-588 is a prognostic marker in gastric cancer. Aging 13: 2101-2117, 2020.

56. Liu Z, Mo H, Sun L, Wang L, Chen T, Yao B, Liu R, Niu Y, Tu K, $\mathrm{Xu} \mathrm{Q}$ and Yang N: Long noncoding RNA PICSAR/miR-588/EIF6 axis regulates tumorigenesis of hepatocellular carcinoma by activating PI3K/AKT/mTOR signaling pathway. Cancer Sci 111: 4118-4128, 2020

57. Yu M, Zhang X, Li H, Zhang P and Dong W: MicroRNA-588 is downregulated and may have prognostic and functional roles in human breast cancer. Med Sci Monit 23: 5690-5696, 2017.

58. Zhao N, Lin T, Zhao C, Zhao S, Zhou S and Li Y: MicroRNA-588 is upregulated in human prostate cancer with prognostic and functional implications. J Cell Biochem: Oct 5, 2017 (Epub ahead of print)

59. Zhou X, Xu M, Guo Y, Ye L, Long L, Wang H, Tan P and $\mathrm{Xu}$ M: MicroRNA-588 regulates invasion, migration and epithelial-mesenchymal transition via targeting EIF5A2 pathway in gastric cancer. Cancer Manag Res 10: 5187-5197, 2018.

60. Shan Z, Shakoori A, Bodaghi S, Goldsmith P, Jin J and Wiest JS: TUSC1, a putative tumor suppressor gene, reduces tumor cell growth in vitro and tumor growth in vivo. PLoS One 8: e66114, 2013.

61. Zhang R, Yu W, Liang G, Jia Z, Chen Z, Zhao L, Yuan Y, Zhou X, $\mathrm{Li}$ D, Shen $\mathrm{S}$, et al: Tumor suppressor candidate 1 suppresses cell growth and predicts better survival in glioblastoma. Cell Mol Neurobiol 37: 37-42, 2017.

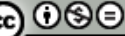

This work is licensed under a Creative Commons Attribution-NonCommercial-NoDerivatives 4.0 International (CC BY-NC-ND 4.0) License. 